\title{
Demand Setting in Internet of Intelligences based on the Factor Vine
}

\author{
Jun Guo ${ }^{1,2}$, Chongfu Huang ${ }^{1,2, *}$, Peizhuang Wang ${ }^{3}$ \\ ${ }^{1}$ Key Laboratory of Environmental Change and Natural Disaster, Ministry of Education of China, Beijing \\ Normal University, Beijing 100875, China \\ ${ }^{2}$ Academy of Disaster Reduction and Emergency Management, Ministry of Civil Affairs \& Ministry of \\ Education, the Peoples' Republic of China, Beijing 100875, China \\ ${ }^{3}$ Institute of Intelligence Engineering and Mathematics, Liaoning Technical University, Fuxin 123000, China
}

\author{
基于因素藤的智联网需求定制模式 \\ 郭君 ${ }^{1,2}$, 黄崇福 ${ }^{1,2, *}$, 汪培庄 ${ }^{3}$ \\ ${ }^{1}$ 北京师范大学环境演变与自然灾害教育部重点实验室, 北京 100875 , 中国 \\ 2 民政部/教育部减灾与应急管理研究院, 北京 100875 , 中国 \\ ${ }^{3}$ 辽宁工程技术大学 智能工程与数学研究院, 辽宁阜新 123000 , 中国
}

\begin{abstract}
In this paper, the factor space theory is introduced to Internet of Intelligences (IOI). The factor vine is used as the theoretical basis for the demand setting in IOI so that the clients could clearly express their problems and the agents could provide effective information. In this way, the operation efficiency of IOI could be improved. A model of multiple IOI is designed to process multi-structure and multi-source information online based on factor vine.

Keywords: Internet of intelligences; Demand setting; Factor space; Factor vine; Multiple Internet of intelligences

摘要

本文将因素空间理论引入智联网的研究, 探讨使用因 素藤作为智联网需求定制的理论依据, 以期帮助使用 智联网的客户能清晰表达问题, 使智能体更有针对性 地提供有效信息, 提高智联网的运行效率。此外, 针 对基于因素藤的需求定制, 本文设计了一种复式智联 网模型, 实现对多层次、多来源信息的在线处理。 关键词: 智联网; 需求定制; 因素空间; 因素藤; 复 式智联网
\end{abstract}

*通讯作者: 黄崇福, hchongfu@bnu.edu.cn

\section{1. 引言}

20 多年来, 互联网帮助人们解决了信息共享和交互, 颠覆了许多传统的商业模式。在智能化突飞猛进的今 天, 互联网将帮助人们集小智慧为大智慧, 形成一个 个全球脑,解决复杂多变的问题, 新的产业呼之欲出。

然而, 传统数学方法难以担当智慧数学的大任, 从线性代数体系到微积分体系, 从概率统计到模糊集 合论, 均不是为研究互联网中的复杂现象而提出的。

人的认识论是对客观事物的反映论, 互联网上的 智慧系统必须能描述复杂多变的客观事物。因素既然 是事物描述的关键, 也就是描述人脑智能活动的关 键。理论上讲, 因素空间的数学理论提供了认知各个 环节的数学描述, 目前正需要在实践中来充实提高。

在互联网大潮中产生的智联网, 已经有了一系列 的实践。这种由智能体、互联网和模型等三元体构成 的, 以期集小智慧为大智慧而实现问题的智能分析和 解答的网络平台[1], 却在模型这个环节遇到了极大 的挑战。其中, 需求者的问题定制, 就是一个突出的 问题。

需求定制是智联网进行解答问题的触发点。文 [2] 在设计第一个智联网平台上进行温州地区开展水产 养殖台风灾害保险可行性的案例研究时, 选用近几年 台风频率、强度的发展趋势、温州水产养殖业发展现 状及趋势等问题要点, 不同的智能体提供对应的经验 信息, 这都是根据保险需求而定的。文[3]在研究智 联网驱动的社区风险雷达时, 选取的则是风险事件的 
危害度、紧急度、事件类型、相关描述作为问题要点。 文[4]在应用其提出的属性拼图智联网研究城市道路 暴雨内涝灾害风险时, 使用降雨过程数据、易涝点分 布数据、风险承受体等要点。上述研究均根据各自的 研究问题选择了相应的问题要点, 然而并没有给出需 求定制中问题要点设置的理论依据。

以分析的要点、以因素为质根的因素空间理论, 致力于为思维过程、人类知识提供数学描述框架, 已 经在概念生成、因果归纳、正逆推理、综合评价、变 权决策、信息融合等方面形成了算法[5], 并且得到 了广泛的应用 $[7,8,9]$ 。利用因素空间来考虑智联网解 决问题时, 先要思考主要任务和目标是什么? 围绕目 标涉及的因素有哪些, 它们之间的关系是怎样的? 该 问题涉及的上位概念什么, 上位概念能划分出哪些新 概念? 在收集到的因素信息的基础上, 为实现问题的 解决, 如何进行概念生成、因果关系、综合评价等? 上述一系列问题正是需求定制的设置大纲。需求定制 是必须适应知识的内在结构, 因素空间是知识和认知 的数学理论, 因素空间为需求定制提供了直接的理论 依据。

一个因素空间主管一个认知单元, 多个因素空间 链接成一个因素藤。智联网的需要定制不是孤立的一 个认知单元而是它们所形成的层次组合。要发展因素 藤的理论来适应多层次的定制需求, 这是作者们所要 特别强调的思想。本文将以因素藤作为智联网中需求 定制问题的理论框架, 首先介绍智联网中需求定制的 任务, 其次说明了因素藤理论, 之后提出复式智联网 模型的信息处理模型。

\section{2. 智联网中的需求定制}

智联网模型是对通过网络、借助智能体实现在线解答 问题的一个抽象, 将通过网络连接起来的能够解决问 题的对象看做智能体, 并通过模型将每个智能体对问 题的解答进行分析、综合等, 实现问题的最终优化解 决。

智联网实现问题在线解决的一般流程是根据研 究的问题进行需求定制, 之后不同的智能体提供相应 要点的信息, 继而通过模型处理信息, 最后得到问题 的优化解答 [2] (图1)。其中, 需求定制是指需要在 智联网平台上解决某一问题的需求者根据关注的问 题 (如前所述, 通常为综合、抽象、复杂的问题), 在平台上设置具体的问题要点 (即具体、直接、简单 的问题), 供智能体在平台上查看并提供相应的信息。 需求定制中的问题要点决定了智联网中信息流的内 容, 合理、恰当的问题要点设置是充分表达待解决问
题的基本任务, 也是保证问题有效解决获得相应信息 的基本需求, 有效、合理的需求定制是智联网实现智 能化的基础。

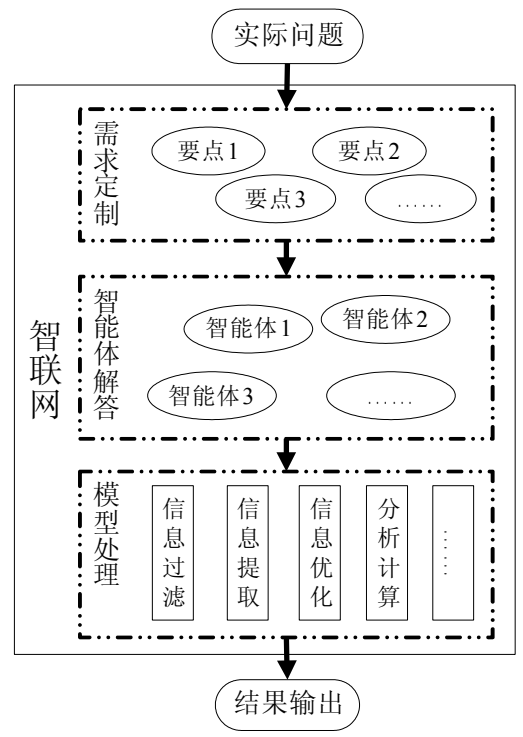

图1. 智联网工作的流程图

在进行需求定制时, 需要围绕待解决的问题分析 与之相关的因素, 分析与问题直接相关的上位概念和 与智联网直接相关的子概念, 给出两者之间的中间概 念, 最后根据上述概念和因素围绕待解决问题设置一 系列问题点。而概念及其对应的因素正构成一个个因 素空间, 因此智联网中的需求定制归根结底是按照因 素空间理论围绕待解决问题进行专业领域知识框架 的建立过程, 研究不同因素空间之间关系的因素藤模 型为该过程提供了算法依据。

\section{3. 基于因素藤的需求定制模式}

\section{1. 因素空间理论}

随着计算机、互联网技术的发展以及人工智能、信息 科学的兴起, 信息的海量化和自由表达形式为信息的 处理、知识的发现提出了新的挑战, 由此构成了智能 数学的使命。在此背景下, 致力于知识发现的因素空 间理论与形式概念分析、粗粘集理论共同构成了智能 数学的三个代表分支。由于其以属性值的质根即因素 为研究对象, 因素空间理论为信息描述提供了统一的 普适性坐标框架, 化解了其他两大分支在知识发现的 


\section{Risk Analysis and Crisis Response in Big Data Era (RAC-16)}

过程中面临的 NP-hard 困境[6,7], 因此其作为智能数 学的理论基础作用将愈发突出。

在因素空间理论中, 因素是属性的统帅, 是信息 科学的质根 [6], 类比于基因对于生命科学的意义, 而更广泛地基因可以看做因素的一个特例。

在数学上, 因素是一个映射, 描述事物或现象与 其某一属性值之间的额对应关系，表示为对象描述的 一个坐标维度。多个因素综合交叉构成对象描述的一 种普适坐标框架，即对象集的因素空间。在因素空间 里, 每一个对象可以根据其诸因素的取值而确定为统 一坐标框架中的一个点。例如, 小麦因干旱造成的减 产量, 可以由降雨量、灌溉能力、作物生长阶段等多 个因素决定, 确定上述因素便可得到一个确定的减产 量值。再如, 一个人可以由身高、年龄等等因素进行 唯一确定, 表达为诸因素构成的因素空间中的一个 点。

因素空间由描述同一个对象集及其若干个因素 构成, 其形式化表达为:

$$
\psi=(U, X(F)) \text { 为 } U \text { 上的一个因素空间 }
$$

其中: $U$ 为因素描述的事物或现象组成的对象集; $X(F)=\{X(f)\}_{(f \in F)}$ 为对象集在因素 $f$ 的映射值组成的集 合。

\section{2. 构建需求定制框架的因素藤}

在现实世界中, 很显然地, 不是所有因素都能用来描 述同一个问题, 即不同因素的论域可能不同, 也即不 是所有因素都可以放到同一个因素空间中, 因此不同 的论域及其对应的因素集构成了不同的因素空间。然 而, 不同的论域可能由于概念细化或原因要素的分解 造成, 则与之相应的不同因素空间之间也存在着一定 的联系，因素藤便是描述不同因素空间之间的这一关 系图, 是思维分析深入过程的一种数学描述。

对于智联网中的需求定制, 往往待解决的问题是 抽象、综合、复杂的, 需要分析待解决问题对应的上 位概念与智能体可能提供信息的子概念及其中间概 念, 进而设计问题要点, 将问题转换为直接、单一、 简单的子问题, 如何构建子问题便是围绕待解决问题 的一个思维细化过程, 因此因素藤便可以为之提供理 论依据。

事实上, 以往的智联网研究已经是基于因素藤的 思想进行需求定制的。例如, 曾凡雷在城市道路暴雨 内涝风险分析智联网研究中, 需求定制了积水点等风 险源要素的子问题、商铺及车辆等风险承受体要素的 子问题、降雨量及地势等综合环境要素的子问题, 分 别形成各个要素的因素空间, 不同因素空间根据要素 之间的关系而形成层次关系, 构成了城市道路暴雨内
涝风险的因素藤, 相应的智能体提供不同要素的相关 信息, 继而采用属性拼图等处理模型得到最终的风险 情景结果[4]。

再如, 针对某一农作物干旱风险有效期评估的智 联网研究中, 需求定制了气候变化等干旱发生规律变 化的子问题、作物品种及灌溉能力等作物抗旱能力变 化的子问题以及作物干旱风险变化的子问题, 分别形 成了干旱发生规律、作物干旱脆弱性、作物干旱风险 三个因素空间, 基于风险的系统性而得到上述三个因 素空间构成的因素藤, 不同的智能体根据自身经验提 供不同因素空间的信息, 在此基础上综合评估出有效 期值[12]。

因此, 基于因素藤进行智联网的需求定制, 可以 围绕待解决的问题构建分析的因素、确定因素之间的 关系、得到不同层次上的因素空间。其中, 构建的每 个因素空间涉及的对象及其因素集构成因素表 [7], 作为智联网数据库设计的内容依据; 而因素藤表达的 因素空间的上下层关系, 表达了问题分析的深入过 程, 为智联网的信息处理模型提供方法依据。

\section{4. 复式智联网的信息处理模型}

\section{1. 复式智联网}

智联网实现正向智能化的关键在于模型, 这也是智联 网优于常见问答网站的关键。

智联网平台中信息往往有着不同可靠性和结构 化程度, 继而需要不同的处理过程。例如, 来自智能 体的主观经验信息往往采用自然语言描述的文本形 式, 需要进行虚假、恶意信息过滤、有效信息提取的 过程; 针对提取后的有效信息, 仍然为自然语言表述, 需要进行结构化处理的过程, 以便后续的量化分析; 对于有限的定量化的样本数据, 则可以采用数学模型 进行分析, 等等。针对这一问题, 采用信息分层的方 式实现智联网中的信息处理过程, 如此得到的智联网 称为复式智联网 [10]。

复式智联网模型的形式化定义如下[11]:

定义 1 设 $A$ 是一个智能体集合, $N$ 是 $A$ 使用的 一个网络, $M$ 是处理 $A$ 所提供信息的模型, 三元体 $<\mathrm{A}, \mathrm{N}, \mathrm{M}>$ 称为一个智联网, 记为 $\Phi$ 。

同态信息是指来自同样途径、关于同一问题的信 息。例如, 对于某区域的洪水淹没深度估计问题, 不 同人的经验判断信息 (如水没及膝盖位置)、淹没水 深标记线得到的记录信息、洪水动力学情景模拟得到 的信息、对他人经验信息得到的总结信息等, 分别为 不同的同态信息。再如, 吴形在研究智联网驱动的社 
Risk Analysis and Crisis Response in Big Data Era (RAC-16)

区风险雷达中选取了危害度、紧急度、事件类型、相 关描述等不同方面描述社区风险事件, 分别由初始事 件提交者和社区工作评价者进行处理而得到了不同 的同态信息[3]。

从因素空间理论出发, 复式智联网解决的是针对 同一问题的一个因素空间的信息, 例如上述表达积水 深度的信息构成了积水事件的一个因素空间, 描述社 区灾害事件危害度、紧急度、事件类型、相关描述等 构成了社区风险的一个因素空间。而不同的同态信息 是指信息可能来自不同的途径, 导致一个因素空间中 信息的质量水平不同，因此采用分层方式进行处理， 即构成了多层信息处理的复式智联网模型。

复式智联网模型的一个简单处理流程可以为: 针 对某一问题构建其因素空间, 解答者智能体对该问题 的认知能力构成的经验判断层, 继而再次利用智能体 的分析能力构成评估分析层, 再次采用信息扩散等算 法构成的数学模型处理层, 最后得出问题的最终解 答。示意图见图 2。

\section{2. 基于因素藤的深度复式智联网}

如前所述, 智联网中面临的问题往往是综合、复杂、 抽象的, 相应地需求定制设置的问题要点是多样的, 即智联网中不仅包括关于同一问题的信息 (构成同一 个因素空间), 而且包括针对不同问题的信息（构成 不同的因素空间), 也即基于因素藤构建的不同因素 空间关系图。

针对基于因素藤进行的需求定制, 本文提出采用
分 “级” 的方式实复杂问题的在线处理, 即深度复式 智联网模型。

为了定义深度复式智联网, 我们须对智联网中的 模型 $M$ 关于其是否能处理多层次的信息进行分类。

同级信息是指关于某一问题同一层次上的信息, 构成同一个因素空间。例如, 关于农作物干旱风险有 效期问题, 干旱发生规律、作物干旱脆弱性的信息属 于作物干旱风险因素空间里的同级信息, 而作物品 种、种植结构、抗旱能力等因素的信息属于下一级作 物干旱脆弱性因素空间里的同级信息, 气候变化等因 素的信息属于下一级干旱发生规律因素空间里的同 级信息。

同级信息可以包括一种或多种同态信息, 也即同 一因素空间中不同来源的信息。例如，上述关于某区 域的洪水淹没深度估计问题的例子中, 不同人的经验 判断信息、淹没水深标记线得到的记录信息、洪水动 力学情景模拟得到的信息、对他人经验信息总结得到 的信息等不同的同态信息, 均解答的是区域淹没水深 这一层次的问题, 因此属于同级信息。再如, 关于农 作物干旱风险有效期问题, 针对干旱发生规律的同级 信息，可能来自专家评判、全球降水模型、历年降雨 量数据分析等不同处理过程给出, 构成了该级不同的 同态信息。

我们将 $M$ 中处理同级信息的一个过程, 称为 $M$ 的一个级; 如果 $\mathrm{M}$ 中的级数多于 1 , 称为 $M$ 的多个 级。此时, 称 $M$ 是一个多个级。例如, 线性回归模 型是一个单级模型, 而线性回归模型和相关分析组成

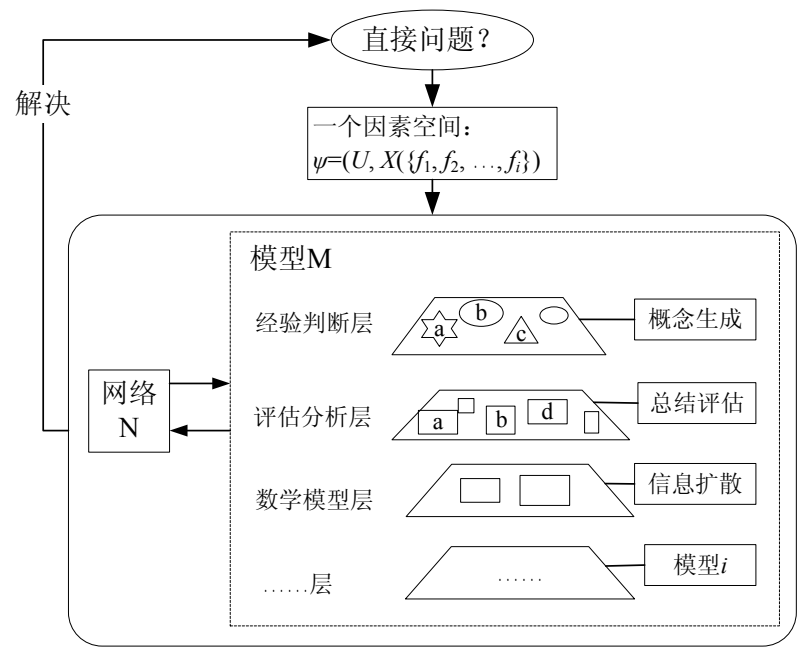

图 2. 多层处理信息的复式智联网示意图。a、b、c、d 构成智联体集 $A$, 通过 网络 $\mathrm{N}$ 连接，所有层共同构成智联网的模型 $\mathrm{M}$ 。 
Risk Analysis and Crisis Response in Big Data Era (RAC-16)

的复合模型, 是一个二级模型。

据此, 我们给出深度复式智联网的形式化定义如 下:

定义 2 设 $\Phi=\angle A, N, M>$ 是一个智联网。如果 $M$ 的是一个多级模型, 称 $\Phi$ 为一个深度复式智联网。

由于定义 2 中设定 $\mathrm{M}$ 的级数多于 1 , 而同级信 息至少包括一种或多种同态信息, 因此在此界定下的 智联网同时属于复式智联网, 因此定义 2 界定的智联 网为深度复式智联网。

深度复式智联网的不同级表征了问题的不同层 次, 形成了不同层次因素空间构成的因素藤。其中问 题划分的层次数决定了级的个数, 相应地称为该级数 的深度复式智联网。例如, 智联网中问题的解决划分 为三个层次, 则可以得到三级深度复式智联网。最简 单的深度复式智联网为二级。深度复式智联网的示意 图如图 3。

\section{5. 结论与讨论}

以网络智能化为目标的智联网解决问题的一般流程 是: 根据待解决的问题进行需求定制, 设置具体的问 题要点, 继而不同的智能体提供相应要点的信息, 在 此基础上通过模型处理得到最终的结果。

由于智联网中要解决的问题往往是综合、复杂、 抽象的, 需求定制通过设置具体要点可以将问题简化 为智能体能够在线提供自身经验信息的简单问题, 合 理的要点设置是问题清晰表达、信息有效获取的前 提。作为智能数学一大分支的因素空间理论, 以信息 的质根即因素为研究对象, 为知识获取、思维分析提 供了数学理论基础。其中, 表达不同因素空间之间关 系的因素藤理论为问题分析逐渐深入的思维过程提 供了描述方法, 致力于描述问题分析要点即因素以及 表达因素之间的关系, 正好为智联网中待解决问题的 要点设置提供理论依据和表达方法, 因此, 本文指出 采用因素藤构建需求定制的问题要点, 不同的因素空

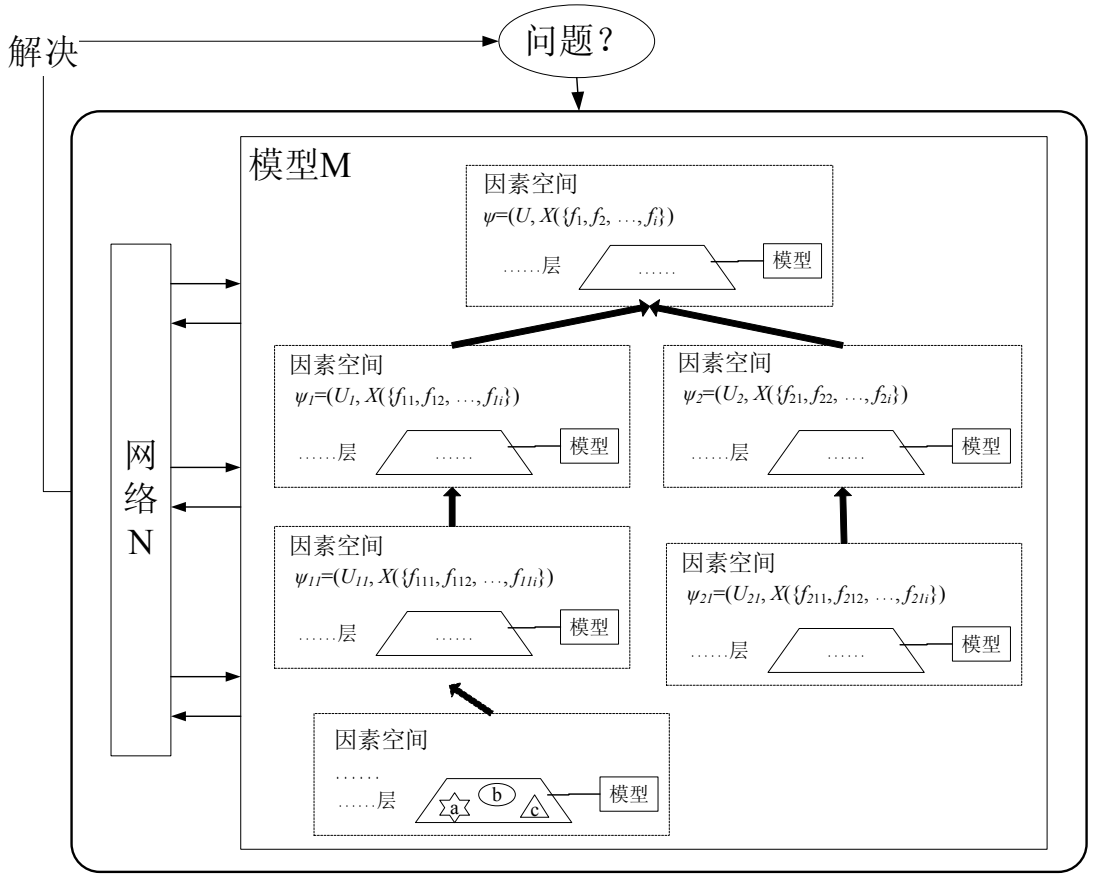

图 3. 基于因素藤的深度复式智联网示意图。 $\mathrm{a} 、 \mathrm{~b} 、 \mathrm{c}$ 构成智联体集 $\mathrm{A}$, 通过网络 $\mathrm{N}$ 连接, 所有级共同构成智 联网的模型 $M$ 
Risk Analysis and Crisis Response in Big Data Era (RAC-16)

间对应于问题的一个分析要点，各个因素空间间的关 系对应于问题的不同层次。

智联网中的信息处理模型是实现问题智能化解 决的关键, 针对基于因素藤的需求定制得到的关于不 同层次的信息, 为了实现上述不同层次信息处理, 提 出了通过将信息分级的深度复式智联网信息处理模 型, 问题的分解层次对应于深度复式智联网的级数。 本文从理论上探讨了基于因素藤的需求定制模 式及其相应的深度复式智联网信息处理模型, 简要介 绍了相关概念和模型, 初步指出了因素空间理论在智 联网中的应用价值, 后续将深入探讨因素运算等在其 的作用, 继而得到更加具体的深度复式智联网模型。 此外, 智联网模型的检验在于其平台的设计实现以及 案例的实践应用, 因此后续工作将从上述方面开展。

\section{Acknowledgements}

This study was supported by National Natural Science Foundation of China (No. 41471426).

\section{致谢}

本研究得到了国家自然科学基金项目(41471426)的 资助。

\section{参考文献}

[1] C.F. Huang, Internet of intelligences in risk analysis for online services, Journal of Risk Analysis and Crisis Response, 2011, 1(2): 110-117.

[2] 艾福利，自然灾害风险分析智联网服务平台构
建与应用研究, 北京师范大学博士学位论文, 2013.

[3] 吴殁, 智联网驱动的在线风险雷达在社区应急 管理中的应用研究, 北京师范大学硕士学位论 文, 2015.

[4] 曾凡雷, 属性拼图智联网风险分析方法及其服 务平台的构建与应用研究, 北京师范大学博士 学位论文, 2016.

[5] 汪培庄. 因素空间与数据科学. 辽宁工程技术大 学学报: 自然科学版, 2015, 34(2): 273-280。

[6] 刘海涛, 包研科, 郭嗣琮, 何华灿, 何平. 因素 空间发展评述(待投稿).

[7] 汪培庄. 因素空间与因素库. 辽宁工程技术大学 学报: 自然科学版, 2013, 32(10): 1-8.

[8] 崔铁军, 马云东. 基于因素空间的煤矿安全情况 区分方法的研究. 系统工程理论与实践, 2015, 35(11): 2891-2897.

[9] 柴杰, 江青茵, 曹志凯 \& 周华. 基于因素空间 变权理论的工业故障诊断. 厦门大学学报 (自然 科学版) , 2002, 41(2): 448-452.

[10] C.F. Huang, Multiple Internet of intelligences for risk analysis, Journal of Risk Analysis and Crisis Response, 2014, 4(2): 61-71.

[11] C.F. Huang, Internet of intelligences can be a platform for risk analysis and management, Human and Ecological Risk Assessment, 2015, 21(5): 1395-1409.

[12] 郭君, 赵思建. 自然灾害风险的有效期及其评估, 辽宁工程技术大学学报: 自然科学版, 2016, 35(9): 983-988. 